Elsevier required licence: (C) $<2020>$. This manuscript version is made available under the CC-BY-NCND 4.0 license http://creativecommons.org/licenses/by-nc-nd/4.0/

The definitive publisher version is available online at

[https://www.sciencedirect.com/science/article/pii/S0921453420302410?via\%3Dihub] 


\title{
Modelling analysis of periodically arranged high-temperature superconducting tapes
}

\author{
Jianxun Jina, Runtao Zhang ${ }^{\mathrm{a}}$, Zhiwei Lin ${ }^{\mathrm{b}, \mathrm{c}}$, Youguang Guo ${ }^{\mathrm{b}}$, Jianguo Zhu ${ }^{\mathrm{d}}$, Xiaoyuan Chen ${ }^{\mathrm{e}}$, Boyang Shen ${ }^{\mathrm{f}, *}$ \\ ${ }^{a}$ School of Electrical and Information Engineering, Tianjin University, Tianjin 300072, China \\ ${ }^{b}$ Faculty of Engineering and Information Technology, University of Technology Sydney, Sydney NSW2007, Australia \\ ${ }^{c}$ State Key Laboratory of Reliability and Intelligence of Electrical Equipment, Hebei University of Technology, Tianjin 300130, China \\ ${ }^{d}$ School of Electrical and Information Engineering, University of Sydney, Sydney NSW4334, Australia \\ e School of Engineering, Sichuan Normal University, Chengdu 610101, China \\ ${ }^{f}$ Electrical Engineering Division, Department of Engineering, University of Cambridge, Cambridge CB3 OFA, United Kingdom \\ *Corresponding authors: Boyang Shen. \\ E-mail addresses: bs506@cam.ac.uk (B. Shen).
}

\begin{abstract}
The operation of a high-temperature superconducting (HTS) device is strongly related to its electromagnetic fields and conditions. It is necessary to study the electromagnetic interaction of proximity HTS tapes integrated such as placed in the horizontal or vertical direction, which is important for the design and optimization of HTS devices. This paper presents a numerical model of periodically arranged HTS tapes using the finite element method (FEM). The numerical method has been developed to build the equivalent periodical arrangement of infinite HTS tapes and applied to simulate two basic arrangements, horizontal and vertical periodical arrangements. The magnetic field and current distributions of HTS tapes by periodical arrangements have been investigated. The effects on critical current with different arrangements have been illustrated. It is found that the $\mathrm{Y}$-stack can reduce the current carrying capacity while the $\mathrm{X}$-array can increase that in comparison to a single tape. The differences between the FEM results and analytical models have been analysed and also compared with the Norris model. The two mathematical models derived for AC loss prediction have shown higher accuracy than the existing analytical models. This study presents an effective method to optimize the electromagnetic fields, estimate the AC loss, and shows the phenomenon of central tapes in an HTS array, which could be potentially used in performance analysis of superconducting devices, e.g. HTS transformers.
\end{abstract}

Keywords: High-temperature superconductor (HTS), HTS tape, HTS electromagnetic characteristics, Finite element method, Periodical arrangement of HTS tapes, Proximity effect, Critical current, AC loss.

\section{Introduction}

The characteristics of zero resistance below the critical temperature enable a revolutionary application prospect of high-temperature superconductors (HTS) [1]. With regard to the HTS application study, simulation analysis has been applied to verify HTS tapes such as for their electromagnetic properties [2]. In practical engineering applications, multiple superconducting tapes are usually periodically stacked to improve the capacity of current transmission and obtain a stronger magnetic field to meet the actual application needs [3]. When the HTS tapes are closely arranged, the magnetic field around an HTS tape will affect its adjacent tapes, i.e. there is a proximity effect. In a complex superconducting system, the HTS components are usually subject to strong magnetic fields, and $\mathrm{AC}$ losses are generated if the magnetic fields are time-varying. Therefore, modelling of the magnetic field and current density distribution in practice is critical for improving the performance of HTS devices so as to reduce the $\mathrm{AC}$ loss and improve the current carrying capacity [4].

Several theoretical calculations of periodical arrangements have been presented for analyzing the magnetic fields in HTS thin tapes with different arrangements [5-8]. However, these models ignored the thickness and anisotropy of the tape and assumed the critical current is constant. The magneto-optical imaging (MOI) technique was used to visualize the magnetic flux density to obtain the real magnetic field distribution in HTS tapes, and the horizontal arrangement of HTS tapes has been studied $[9,10]$, but the magnetic field distribution of the vertical arrangement could not be completely observed due to the limitations of MOI technique [11]. Alternatively, simulations based on the finite element method (FEM) are applied widely in superconductivity research [12-15], FEM analyses have been carried out on the current density of a single tape and the $\mathrm{AC}$ losses in the horizontal and vertical arrangements [16-20]. With only two or three tapes, these basic FEM models cannot simulate the real magnetic field and current distribution of periodically arranged HTS tapes in practice, and they also can not verify or modify the analytical models, which are often used for loss estimation. Therefore, it is important to accurately model the actual magnetic field distribution, and find out the difference between its actual value and analytical solution, in order to estimate the AC loss 
and optimize the performance of HTS devices.

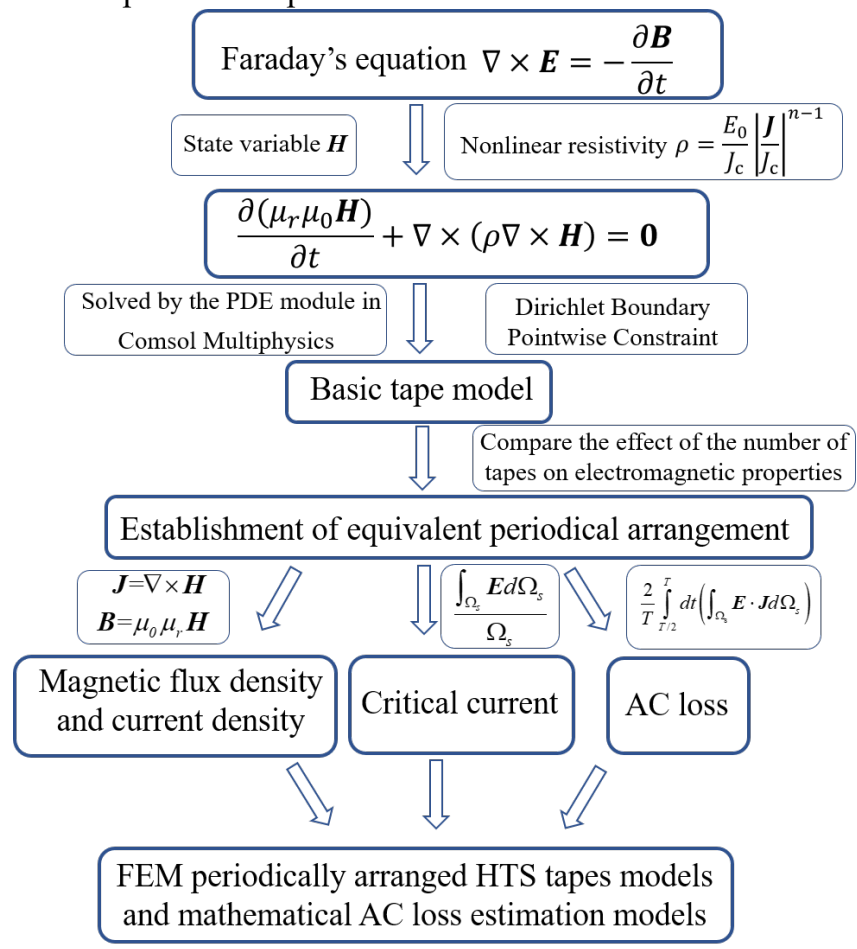

Fig. 1. Flowchart of analysis for periodically arranged HTS tapes.

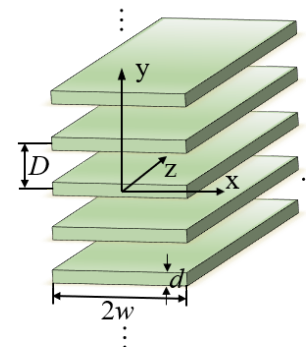

(a)

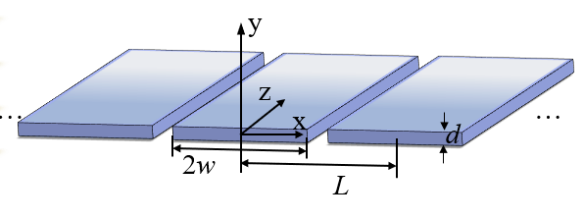

Fig. 2. (a) Arrangement of HTS tapes in Y-stack. (b) Arrangement of HTS tapes in X-array.

In this paper, a numerical model is developed and presented for analyzing the periodically arranged HTS tapes, which is more accurate than the models mentioned above. This paper is organized as follows. Section 2 presents the numerical method of periodically arranged HTS tapes in detail, and an introduction of an analytical model. Section 3 proposes an effective method to establish the model of periodical arrangement in simulation. The magnetic field distribution, critical current, and AC loss by two periodical arrangements are studied in Section 4. The flowchart of analysis for periodically arranged HTS tapes is shown in Fig. 1. These new results would be beneficial to the future design of HTS devices.

\section{Analysis methods of periodical arrangement}

The arrangements of HTS tapes can be simply classified into the $\mathrm{Y}$-stack and $\mathrm{X}$-array. The $\mathrm{Y}$-stack arrangement in this paper is defined as an infinite number of long tapes stacked along the y-axis with a regular distance $D$. The $\mathrm{Y}$-stack is identical to tape-stack investigations with trapped field and other electromagnetic analysis in several certain conditions, as reported in [21-23]. The $X$-array arrangement can be regarded as an infinite number of horizontal tapes aligned in the $\mathrm{x}-\mathrm{Z}$ plane with a regular distance $L$ between the adjacent tapes, as shown in Fig. 2. The width and the thickness of each tape are defined as $2 w$ and $d$, respectively.

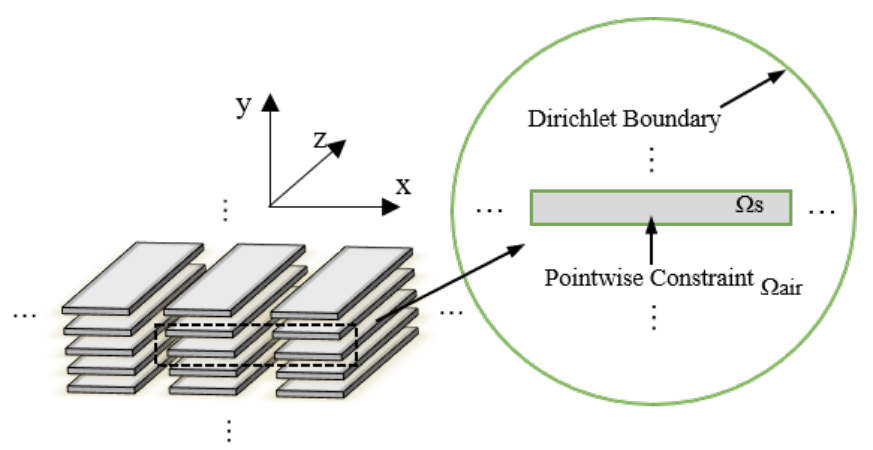

Fig. 3. A numerical model for HTS tapes.

\subsection{Numerical method}

For the proposed geometry as shown in Fig. 3, the 2-D $\boldsymbol{H}$ formulation is derived to study the electromagnetic field distribution and AC loss of HTS tapes numerically by using COMSOL Multiphysics. The 2-D $\boldsymbol{H}$-formulation consists of the Faraday's law (1), Ampere's law (2), Ohm's law (3) and constitutive law (4) [24] as the following:

$$
\begin{gathered}
-\frac{\partial \boldsymbol{B}}{\partial t}=\nabla \times \boldsymbol{E} \\
\boldsymbol{J}=\nabla \times \boldsymbol{H} \\
\boldsymbol{E}=\rho \boldsymbol{J} \\
\boldsymbol{B}=\mu_{0} \mu_{r} \boldsymbol{H}
\end{gathered}
$$

where $\boldsymbol{E}$ is the electrical field strength, $\boldsymbol{J}$ is the current density, $\rho$ is the resistivity, $\mu_{r}$ is the relative permeability and $\mu_{0}$ is the permeability of free space. The power-law relationship is employed for the resistivity in superconducting layers as:

$$
\rho=\frac{E_{0}}{J_{c}(B)}\left|\frac{J_{z}}{J_{c}(B)}\right|^{n-1}
$$

where $n$ is the power-law parameter, and $\rho$ is defined as $1 \Omega \cdot \mathrm{m}$ in the air region [25]. The critical electrical field strength $E_{0}$ is set to $10^{-4} \mathrm{~V} / \mathrm{m}$.

Due to the anisotropy of HTS tape under an external magnetic field, the magnetic field and current distributions in the tape will be greatly affected by the magnetic fields generated by the other tapes. The anisotropy of the critical current density can be expressed as a function of the magnetic field distribution as:

$$
J_{c}(B)=\frac{J_{c 0}}{\left(1+\frac{\sqrt{\beta^{2}\left|B_{\mu /}\right|^{2}+\left|B_{\perp}\right|^{2}}}{B_{0}}\right)^{\alpha}}
$$

where $\alpha, \beta$, and $B_{0}$ are material parameters, $J_{\mathrm{c}}(B)$ is the field- 
dependent critical current density, $J_{\mathrm{c} 0}$ is the critical current density with the self-field and $77 \mathrm{~K}, B / /$ and $B_{\perp}$ are respectively the horizontal and the perpendicular magnetic flux density in the cross sections.

The real dimensions of SuperPower SCS4050 tape with a superconducting layer of $1 \mu \mathrm{m}$ is modelled. Other details are shown in Table I. The data on the central axis is taken as the result of the tape. As the SuperPower SCS4050 tapes have a non-magnetic substrate, there is no ferromagnetic loss. The $\mathrm{AC}$ losses are simulated at $50 \mathrm{~Hz}$. Since the small amount of eddy-current loss can be ignored, the hysteresis loss in the superconducting layer dominates the total AC losses of HTS tape, and the AC loss in watt per meter of the tape can be calculated by [26]:

$$
P=\frac{2}{T} \int_{T / 2}^{T} d t\left(\int_{\Omega_{\mathrm{s}}} \boldsymbol{E} \cdot \boldsymbol{J} d \Omega_{s}\right)
$$

Table 1

Parameters of the Sample HTS Tape.

\begin{tabular}{cc}
\hline \hline Parameters & Value \\
\hline Width of the tape $2 w(\mathrm{~mm})$ & 4 \\
Thick of the tape $d(\mu \mathrm{m})$ & 1 \\
Critical current self-field $I_{\mathrm{c}}$ at $77 \mathrm{~K}(\mathrm{~A})$ & 99.23 \\
Power-law parameter $n$ & 25 \\
$B_{0}(\mathrm{mT})$ & 42.65 \\
$\alpha$ & 0.70 \\
$\beta$ & 0.29515 \\
\hline
\end{tabular}

\subsection{Analytical solution}

The applied DC transport current and flux distributions in the Y-stack or X-array arrangement can be theoretically solved by using the transformation method as described below [8].

For periodical Y-stack with variable transformations of $\tilde{x}=\frac{D}{\pi} \tanh \left(\frac{\pi x}{D}\right), \tilde{b}=\frac{D}{\pi} \tanh \left(\frac{\pi b}{D}\right)$ and $\tilde{w}=\frac{D}{\pi} \tanh \left(\frac{\pi w}{D}\right)$, the current and magnetic field distributions can be expressed respectively as the following:

$$
\begin{gathered}
J(x)=\left\{\begin{array}{cc}
2 J_{c} \arctan \left(\frac{b^{2}-x^{2}}{\sqrt{w^{2}-b^{2}}}\right)^{1 / 2} & |\tilde{x}|<\tilde{b} \\
J_{c} & \tilde{b}<|\tilde{x}|<\tilde{w}
\end{array}\right. \\
H(x)=\left\{\begin{array}{cc}
0 & |\tilde{x}|<\tilde{b} \\
-H_{0} \frac{\tilde{x}}{|\tilde{x}|} \operatorname{arctanh}\left(\frac{\bar{x}^{2}-b^{2}}{\sqrt{w^{2}-b^{2}}}\right)^{1 / 2} & \tilde{b}<|\tilde{x}|<\tilde{w}
\end{array}\right.
\end{gathered}
$$

where $H_{0}=J_{c} d / \pi$ is the characteristic field strength, and:

$$
b=\frac{D}{\pi} \operatorname{arccosh}\left(\frac{\cosh (\pi w / D)}{\cosh \left(\pi w I_{a} / D I_{c}\right)}\right)
$$

$b$ is defined as the flux front measured from the center of the tape in Y-stack.

For the transport current passing through the periodical Xarray, with the variable transformations $\tilde{x}=\frac{L}{\pi} \tan \left(\frac{\pi x}{L}\right)$,
$\tilde{b}=\frac{L}{\pi} \tan \left(\frac{\pi b}{L}\right)$ and $\tilde{w}=\frac{L}{\pi} \tan \left(\frac{\pi w}{L}\right)$, the calculation formulae of current density distribution and magnetic field distribution are the same as those for the Y-stack (see (8) and (9)). However, the calculation formulae of position $b$ in the $\mathrm{X}$-array is different, and the corresponding position $b$ is as the following:

$$
b=\frac{L}{\pi} \arccos \left(\frac{\cos (\pi w / L)}{\cos \left(\pi w I_{a} / L I_{c}\right)}\right)
$$

Self-field hysteresis loss of a unit length tape in the Y-stack and $\mathrm{X}$-array can be expressed respectively as:

$$
\frac{p_{\perp}}{l}=\frac{\mu_{0}}{\pi^{2}} \omega \frac{I_{c}^{2}}{w^{2}} \int_{b_{\perp}(0)}^{w}(w-x) \times \operatorname{arctanh}\left(\frac{\tanh ^{2}(\pi x / D)-\tanh ^{2}\left(\pi b_{\perp}(0) / D\right)}{\tanh ^{2}(\pi w / D)-\tanh ^{2}\left(\pi b_{\perp}(0) / D\right)}\right) d x
$$

$\frac{p_{\| \prime}}{l}=\frac{\mu_{0}}{\pi^{2}} \omega \frac{I_{c}^{2}}{w^{2}} \int_{b_{l}(0)}^{w}(w-x) \times \operatorname{arctanh}\left(\frac{\tan ^{2}(\pi x / L)-\tan ^{2}\left(\pi b_{l \prime}(0) / L\right)}{\tan ^{2}(\pi w / L)-\tan ^{2}\left(\pi b_{l \prime}(0) / L\right)}\right) d x$

where:

$$
\begin{aligned}
b_{\perp}(0) & =\frac{D}{\pi} \operatorname{arccosh}\left(\frac{\cosh (\pi w / D)}{\cosh \left(\pi w I_{a} / D I_{c}\right)}\right) \\
b_{l /}(0) & =\frac{L}{\pi} \arccos \left(\frac{\cos (\pi w / L)}{\cos \left(\pi w I_{a} / L I_{c}\right)}\right)
\end{aligned}
$$

\section{Equivalent Models of periodical arrangement}

\section{in simulation}

In the theoretical derivation, the method of variable transformations is used to introduce the periodical arrangement. However, it is impossible to set unlimited tapes in the simulation. It is necessary to find suitable conditions equivalent to the periodical arrangement of infinite tapes for accurate and fast simulation.

\subsection{Infinite tapes in Y-stack established in simulation}

Fig. 4 shows the flux distributions (Fig. 4(a)) and current distributions (Fig. 4(b)) of central tape at xoz-plane when a 0.6 $I_{c}$ DC current passes through the Y-stack with different numbers of tapes, $N$. The analytical result is given by solid line while the numerical results are given by dash line. The magnetic field is almost unchanged when $N$ is more than 21 . When the Y-stack carries a $0.6 I_{c} 50 \mathrm{~Hz} \mathrm{AC}$ transport current, the AC loss of central tape is almost unchanged when $N$ is more than 21 as shown in Fig 5. It means that it can be equivalent to an infinite periodical arrangement. Hence, 21 vertically arranged tapes can be taken as the conditions for studying the infinite periodical arrangement in the simulation.

Compared with the analytical result, both have similar trends. The current density is smaller at both edges of the tape, since the anisotropy of HTS tape is taken into account in the 
FEM model. It is demonstrated that the simulation method can be applied to analyze the Y-stack arrangement and multiple excitations, which cannot be observed by the MOI technique.

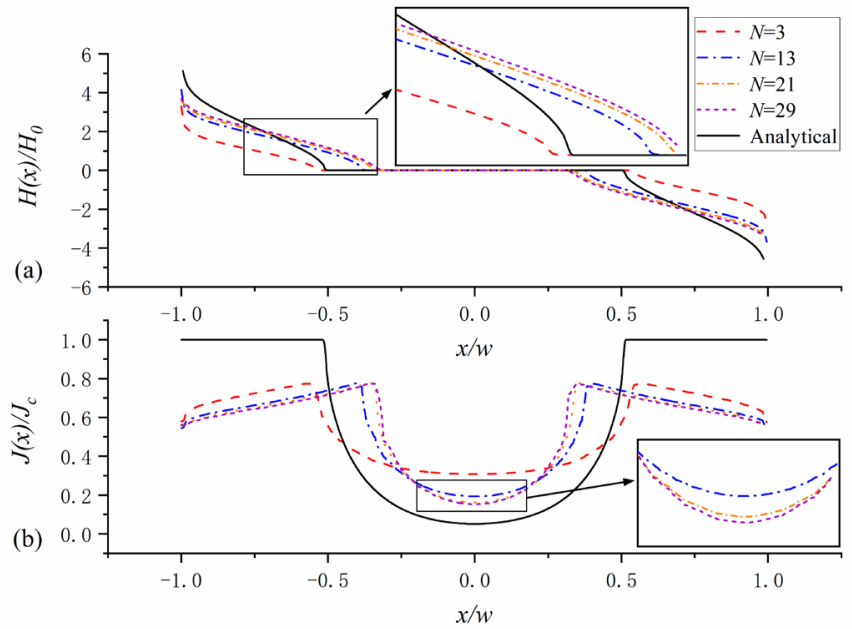

Fig. 4. Normalized flux distributions (a) and current distributions (b) of central tape of Y-stack $(D / w=0.5)$ with the different number of tapes, $N$, when a $0.6 I_{c}$ DC current is applied.

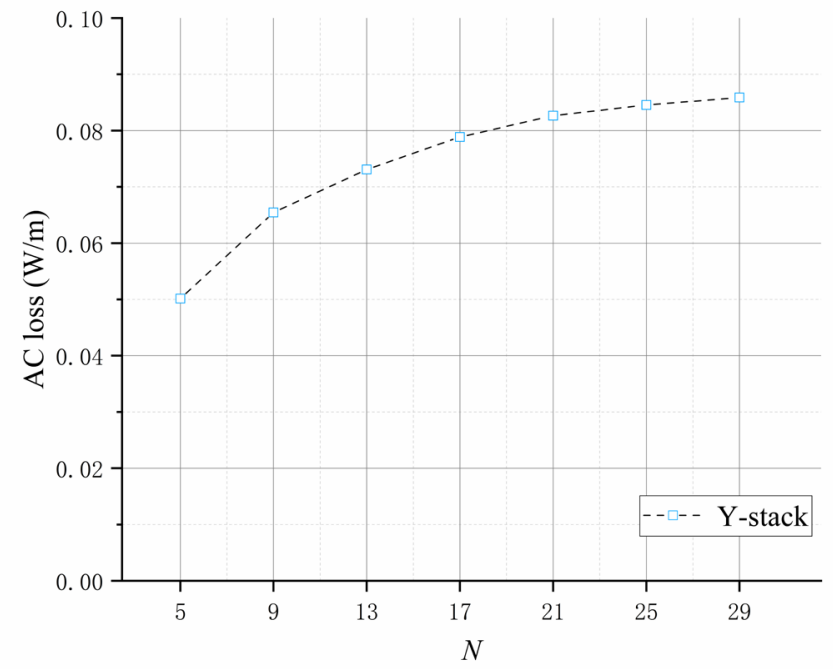

Fig. 5. Numerical AC losses of central tape of Y-stack arrangement $(D / w=0.5)$ with the different numbers of tapes, $N$.

\subsection{Infinite tapes in Y-stack established in simulation}

Fig. 6 shows the flux distributions of central tape arranged in X-array $(L / w=3)$ with the different number of tapes when a $0.6 I_{c} \mathrm{DC}$ current is applied. Fig. 7 shows the numerical losses when a $0.6 I_{c} \mathrm{AC}$ current at $50 \mathrm{~Hz}$ is applied. The results show that the magnetic field and $\mathrm{AC}$ loss of $\mathrm{X}$-array are almost unchanged when $N$ is more than 7 . The difference between the $\mathrm{Y}$-stack and $\mathrm{X}$-array is due to the large width/thickness ratio of the tapes. It is shown that when the number of tapes reaches a certain level, the magnetic field of the central tape is almost unchanged. It means that the central tape can be equivalent to an infinite periodical arrangement. Hence, 7 horizontally arranged tapes can be taken as the conditions for studying the infinite periodical arrangement in the simulation.

Compared with the analytical results, the current density is also smaller at both edges of the tape, since the anisotropy of HTS tape is taken into account. The results are consistent with the results visualized by the MOI technique for the observed magnetic field distribution of single tape and X-array [11].

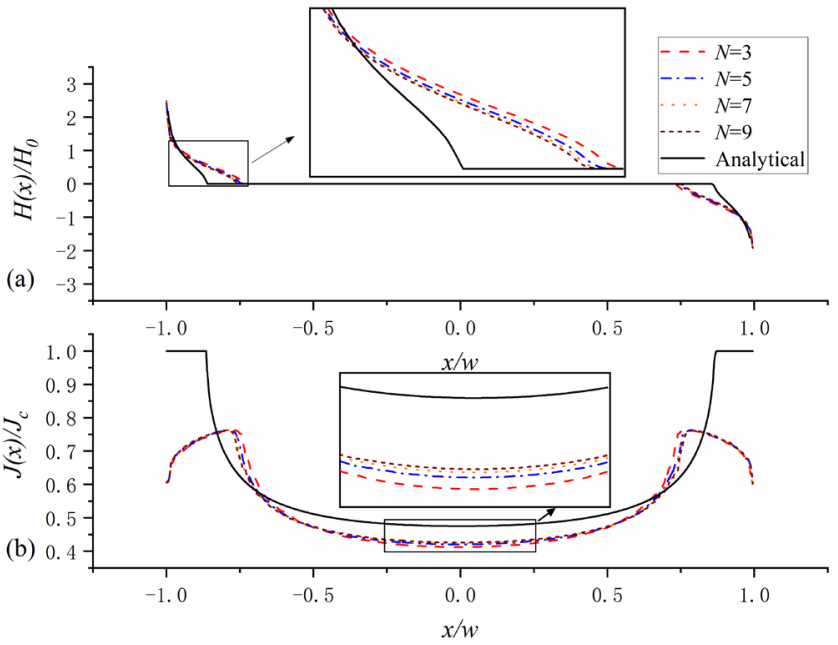

Fig. 6. Normalized flux distributions (a) and current distributions (b) of central tape of X-array $(L / w=3)$ with the different number of tapes, $N$, when a $0.6 I_{c} \mathrm{DC}$ current is applied.

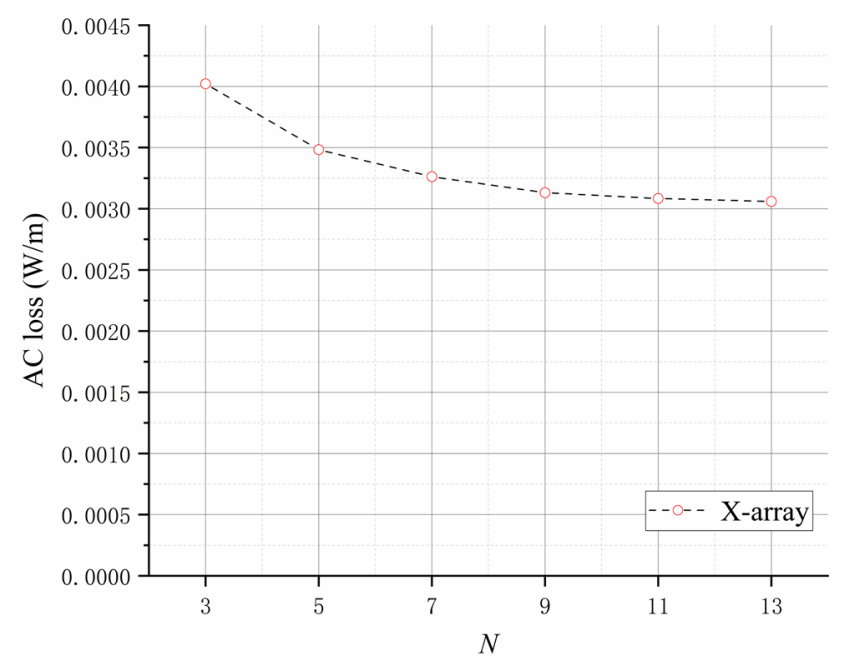

Fig. 7. Numerical AC losses of central tape of $X$-array arrangement $(L / w=3)$ with the different numbers of tapes, $N$.

\section{Results and discussion}

\subsection{Magnetic flux density and current distribution}

As analyzed above, the equivalent periodic arrangement can be carried out when the number of tapes $N$ is 7 for the $\mathrm{X}$-array and 21 for the $\mathrm{Y}$-stack respectively. The following analysis is derived based on this certain situation. Figs. 8-10 illustrate the distributions of the magnetic flux density of the single tape, obtained by using COMSOL simulation, with Y-stack $(D / w=0.5)$ and $\mathrm{X}$-array $(L / w=3)$ having $0.6 I_{c} \mathrm{DC}$ current. The results are significantly different, and the magnetic field of $\mathrm{Y}$ stack is the highest due to the mutual strengthening of the magnetic field of the tapes. The magnetic field of X-array is the weakest because the perpendicular components of the magnetic field generated by other tapes cancel each other, thus effectively shielding the middle tape at central. 


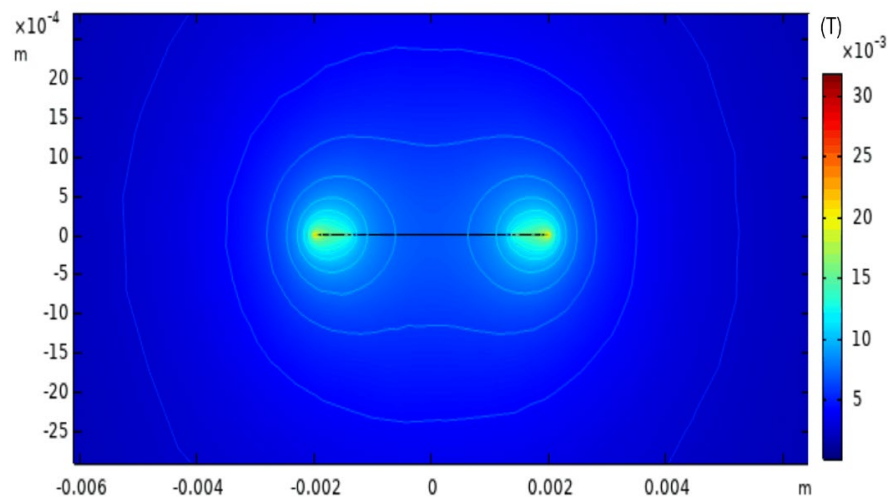

Fig. 8. Magnetic flux density of a single HTS tape when a $0.6 I_{c}$ DC current is applied.

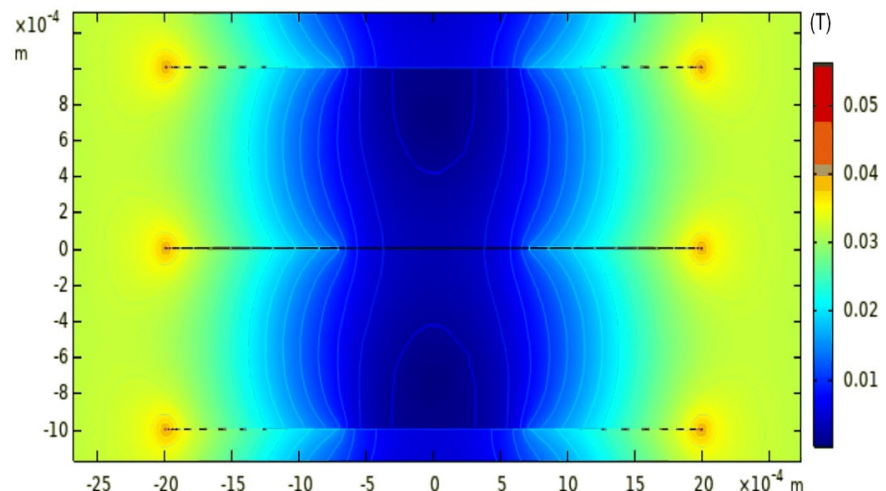

Fig. 9. Magnetic flux density of Y-stack $(D / w=0.5)$ when a $0.6 I_{c} \mathrm{DC}$ current is applied.

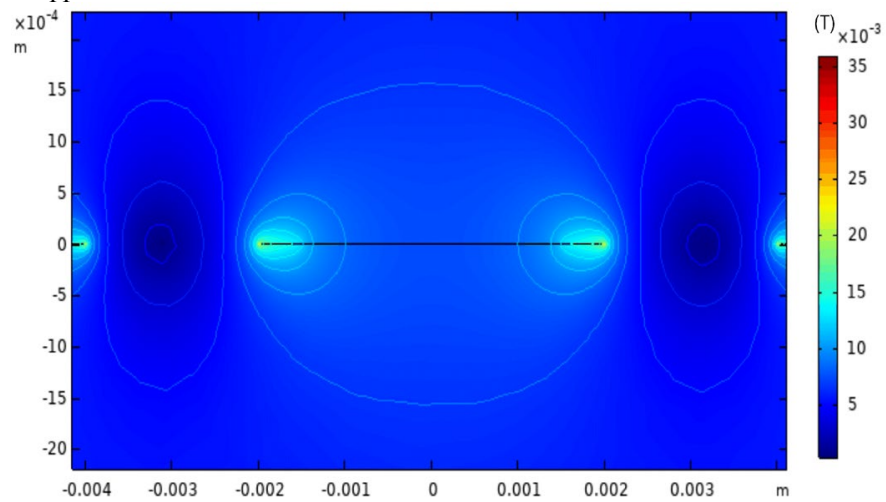

Fig. 10. Magnetic flux density of $X$-array $(L / w=3)$ when a $0.6 I_{c}$ DC current is applied.

Fig. 11 shows the magnetic field and current distributions in single tape, central Y-stack tape $(D / w=0.5)$, and central $\mathrm{X}$ array tape $(L / w=3)$ when $0.2 I_{c} \mathrm{DC}$ current or $0.6 I_{c} \mathrm{DC}$ current is applied. It can be seen that the flux penetrates deeper into the Y-stack than the single tape. In addition, the flux density along the edges arranged in the Y-stack is stronger than that in the single tape. It can be attributed to the superposition of fields near the edges, leading to a reduction in critical current density near the edges. The $\mathrm{X}$-array decreases the flux-filled region compared to a single tape and reduces the edge magnetic field, due to the inverse cancellation of the magnetic field.

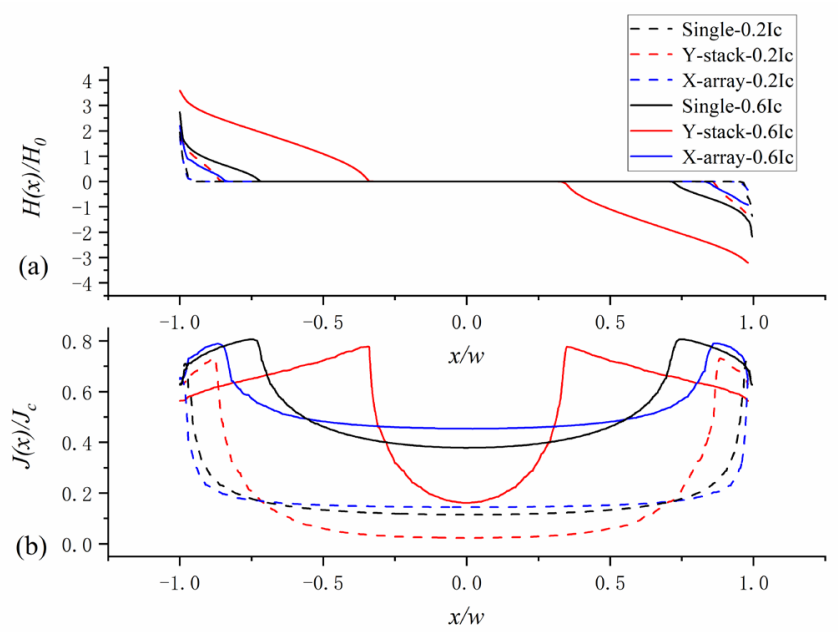

Fig. 11. Normalized magnetic field distributions (a) and current distribution (b) versus $\mathrm{x} / \mathrm{w}$ for single tape, X-array, and Y-stack arrangements when $0.2 I_{c}$ DC current or $0.6 I_{c}$ DC current is applied.

\subsection{Critical current}

Fig. 12 shows the DC current value if the flux penetrations up to $w / 2$ from the edge in the different arrangements. As the distance increases, the $\mathrm{Y}$-stack needs more current to reach half penetration, while $\mathrm{X}$-array needs less current. The result is related to the magnetic field in different arrangements, which is consistent with the above analysis of magnetic field distribution.

Fig. 13(a) shows the current density distribution with $I_{a} / I_{c}=0.75$ and Fig. 13(b) shows the current density distribution when penetration depth reaches half $(b / w=0.5)$ for the single tape, X-array $(L / w=3)$ and $\mathrm{Y}$-stack $(D / w=1)$. When the same amount of current flows, the $\mathrm{Y}$-stack has a larger flux-filled region while the $\mathrm{X}$-array has a smaller flux-filled region. When the penetration depth reaches half $(b / w=0.5)$, the currents flowing through the single tape, $\mathrm{X}$-array and $\mathrm{Y}$-stack are $0.72 I_{c}, 0.77 I_{c}$ and $0.56 I_{c}$, respectively. The X-array appears to be able to carry more current. To verify our ideas, the $I-V$ curves of different arrangements as shown in Fig. 14 are calculated by [27]:

$$
\text { Tape voltage }=\frac{\int_{\Omega_{s}} \boldsymbol{E} d \Omega_{s}}{\Omega_{s}}
$$

The $I-V$ curve of a single tape shows that the critical current is around $100 \mathrm{~A}$, which is consistent with the parameters of the selected tape. Hence, it can be concluded that the model simulates the tape very well.

The Y-stack reduces the critical current compared with the single tape because of the magnetic field enhancement at tape ends. The critical current of Y-stack increases as the distance increases. The X-array increases the critical current compared with the single tape due to the weakening of the magnetic field by the horizontal arrangement. The critical current of X-array decreases as the distance increases. It should be noted that it is impossible to infinitely arrange the tapes in an actual HTS device, and these numerical results can show the phenomenon of central tapes in an HTS array. 


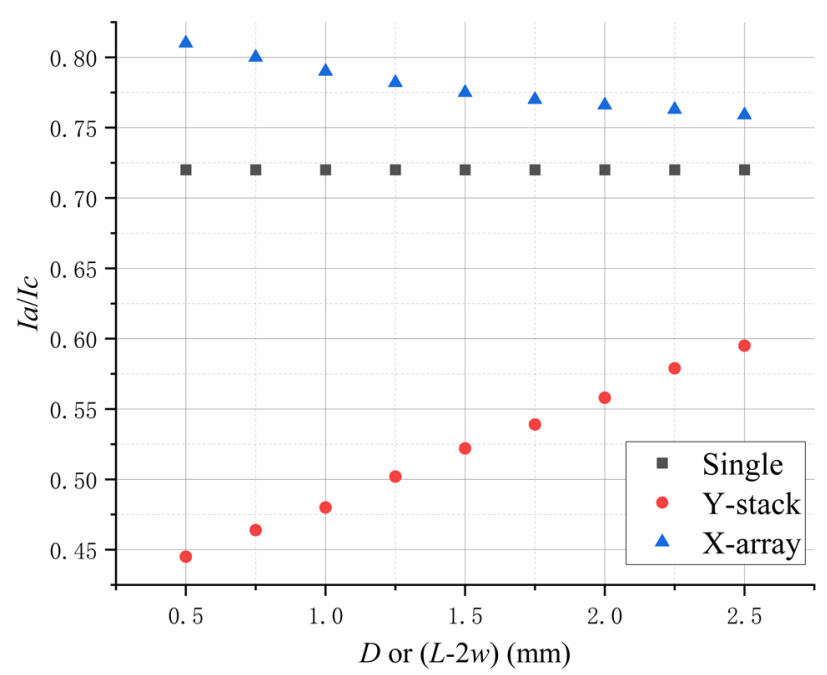

Fig. 12. Half flux penetration attainment of the current $I_{a}$ with different distances.

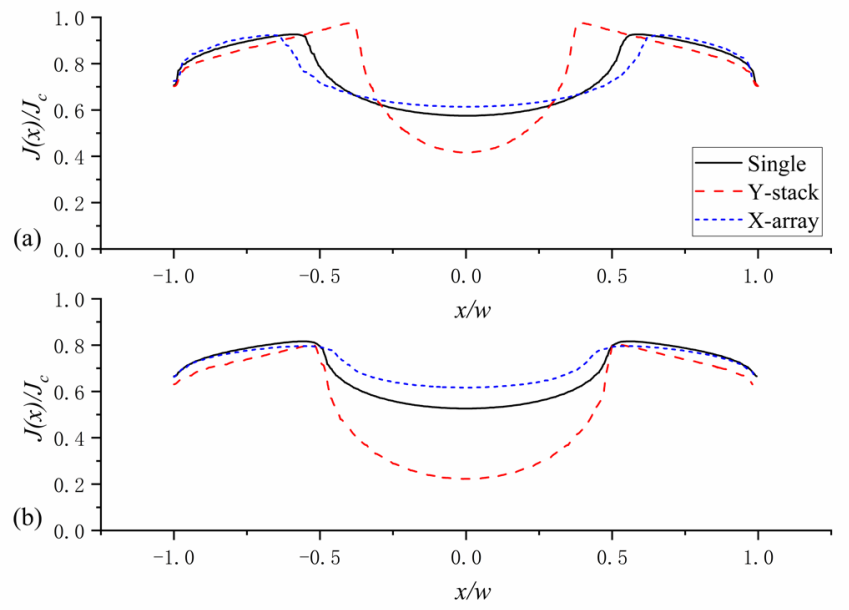

Fig. 13. (a) Current density distribution with $I_{a} / I_{c}=0.75$; (b) Current density distribution with half penetration $(b / w=0.5)$.

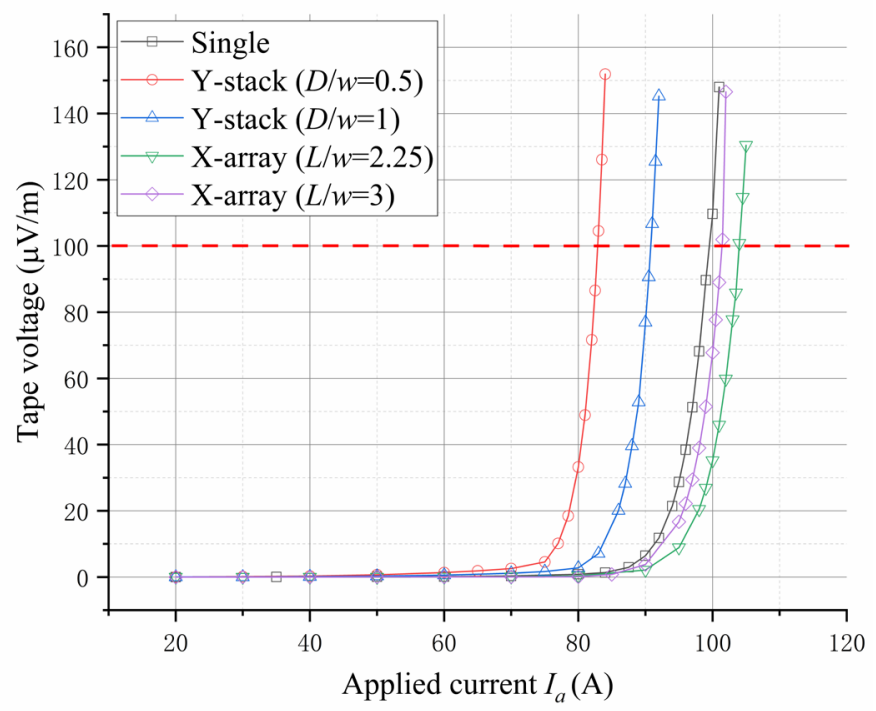

Fig. 14. $I-V$ curves for different arrangements.

\subsection{AC loss}

Fig. 15 shows the AC loss for a Y-stack at $50 \mathrm{~Hz}$ compared with the analytical result. It can be seen that the loss values by both calculations show good agreement when the tapes are arranged closely. However, the difference is significant when the tapes are far apart. When $D / w$ is larger, that is, the tape is approximate to be isolated $(D / w \rightarrow \infty)$, the loss calculated by both methods are in good agreement, and it is also closer to the value calculated by the Norris model [28]. The results show that Y-stack increases the loss. It is consistent with deeper flux penetration and stronger flux density near the edges arranged in the Y-stack compared with the single tape.

Fig. 16 shows the $\mathrm{AC}$ loss for an $\mathrm{X}$-array at $50 \mathrm{~Hz} \mathrm{AC}$. The $\mathrm{X}$-array shows less AC loss compared with the $\mathrm{Y}$-stack arrangement. When distance $L$ approaches $2 w$, the effect of parallel magnetic field $H_{x}$ gradually increases and AC loss will decrease. Compared with the analytical model, the difference between the two methods is great when the distance $L$ is small, and the difference decreases as the distance $L$ increases. When $L / w$ is larger, the tape is closer to an isolated tape $(L / w \rightarrow \infty)$, the simulation results are closer to the value calculated by the Norris model

In order to meet more accurately the needs of loss estimation in practical applications, the empirical expressions for AC loss per unit length with different distances (mm) are proposed respectively as follows:

$$
\frac{p_{\perp}}{l}=a+b \cdot D^{c} \cdot\left(\frac{I_{a}}{I_{c}}\right)^{e}
$$

where constants $a=2.804 \times 10^{-3}, b=0.5718, c=-1.237$, and $e=4.0874$ for a Y-stack, and:

$$
\frac{p_{\| \prime}}{l}=a+b \cdot(L-2 w)^{c} \cdot\left(\frac{I_{a}}{I_{c}}\right)^{e}
$$

where constants $a=1.065 \times 10^{-4}, b=0.0235, c=0.6144$, and $e=4.9663$ for an X-array.

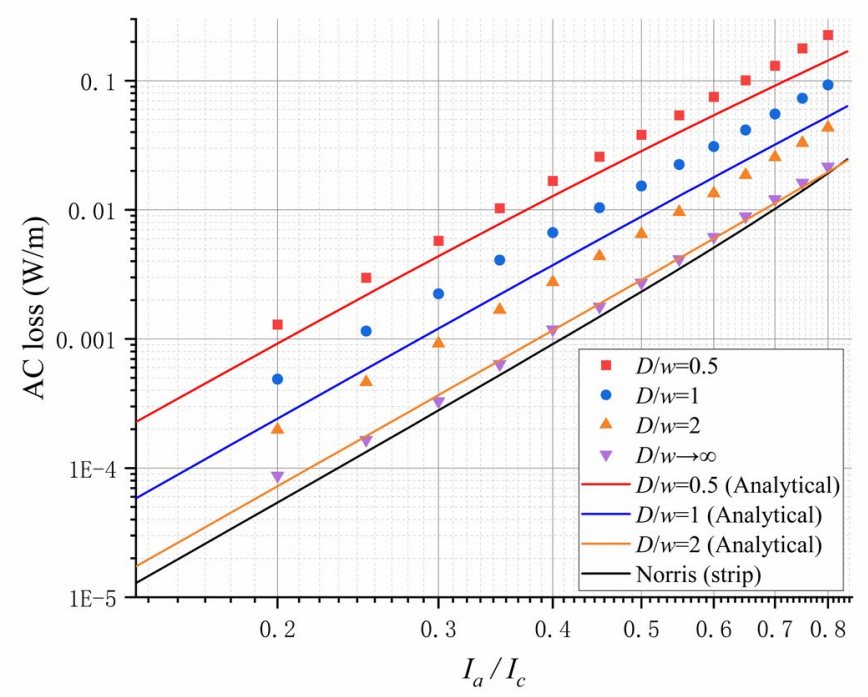

Fig. 15. AC loss per length with different AC current amplitudes $\left(I_{a} / I_{c}\right)$ for a Y-stack. 


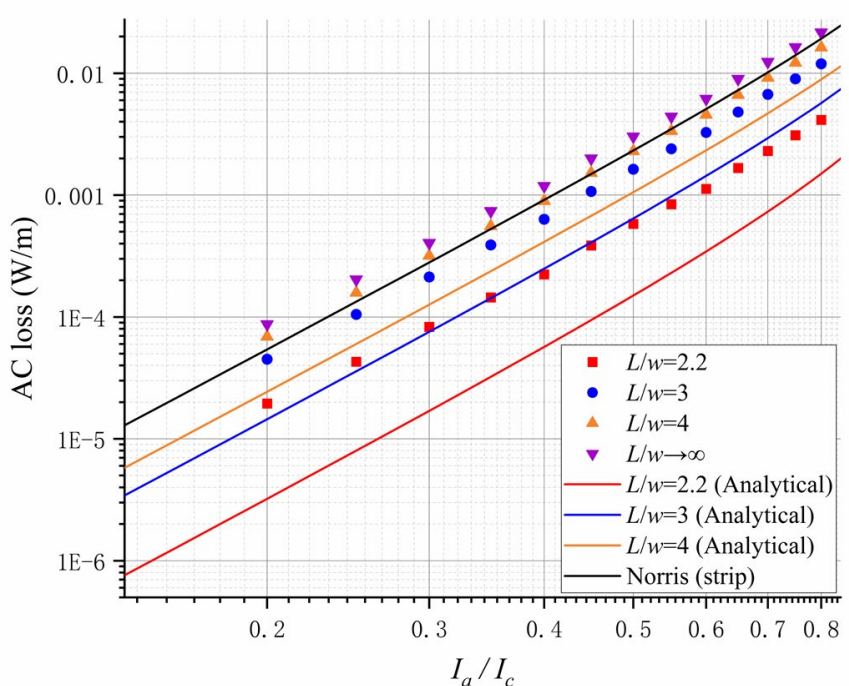

Fig. 16. AC loss per length with different AC current amplitudes $\left(I_{a} / I_{c}\right)$ for an $\mathrm{X}$-array.

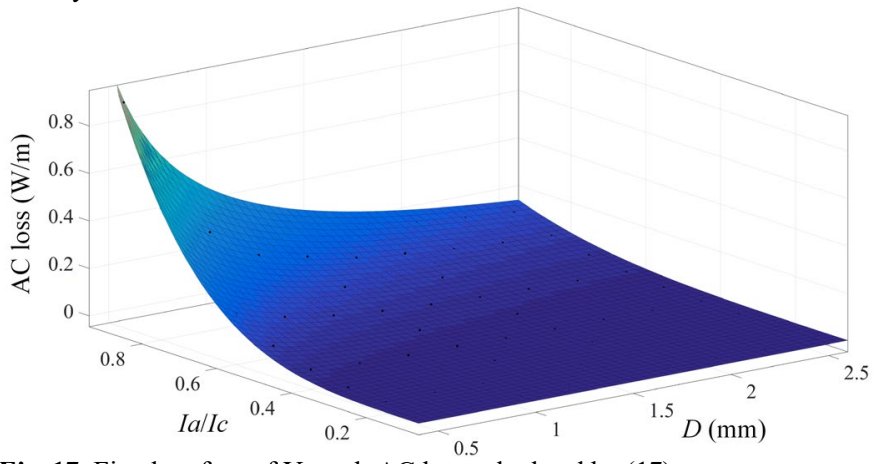

Fig. 17. Fitted surface of Y-stack AC loss calculated by (17).

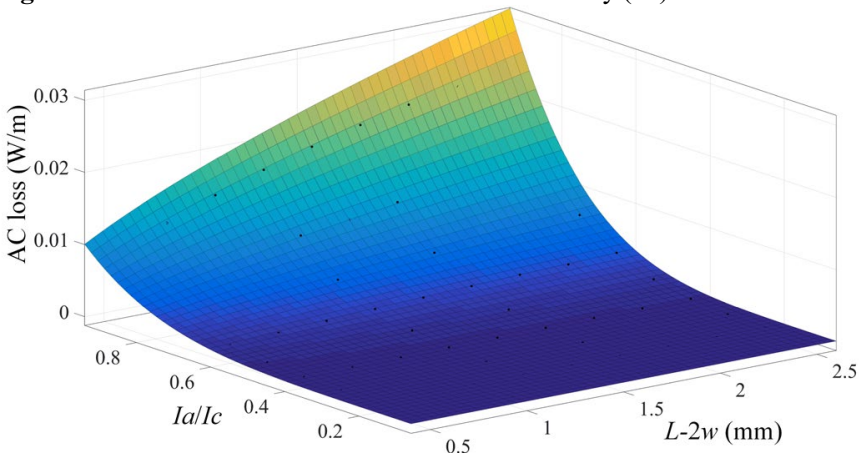

Fig. 18. Fitted surface of $X$-array AC loss calculated by (18).

Figs. 17 and 18 show the results of AC loss per unit length with different distances and currents for a Y-stack and $\mathrm{X}$ array. The AC loss decreases as the distance $D$ increases for a $\mathrm{Y}$-stack, and the AC loss increases as the distance $L$ increases for an $\mathrm{X}$-array. The numerical estimations have good fitting results, which means that they can be used to estimate more accurately the AC loss compared to analytical models.

\section{Conclusion}

A numerical model of periodically arranged HTS tapes is presented using FEM based on the $\boldsymbol{H}$-formulation. Firstly, the method is shown how to realize the periodical arrangement of infinite HTS tapes in simulation. Then, the profiles of the magnetic field and current density are discussed. The flux penetration of Y-stack is deeper than the single tape due to the superposition of fields near the edges, while the $\mathrm{X}$-array decreases the flux-filled region due to the cancellation of the magnetic field. Moreover, the critical current and AC loss are also analyzed from the perspectives of Y-stack and X-array. It can be concluded that the $\mathrm{X}$-array will improve the current carrying capacity, while the Y-stack will reduce that. The AC loss of $\mathrm{Y}$-stack will decrease as the distance increases, whereas the AC loss of $\mathrm{X}$-array will increase. The differences between the FEM results and analytical models are discussed. Both FEM results are higher than those of the analytical model which fail to consider anisotropy and the change of critical current. The loss of the numerical model will eventually approach the value calculated by the Norris model as the distance increases. Two mathematical models for the $\mathrm{AC}$ losses with different distances are proposed for the periodically arranged HTS tapes, which are more accurate to estimate the AC loss compared with the analytical models. These new results would be beneficial to the future design of HTS devices.

\section{References}

[1] J.X. Jin, Y.J. Tang, X.Y. Xiao, B.X. Du, Q.L. Wang, J.H. Wang, S.H. Wang, Y.F. Bi, J.G. Zhu, HTS power devices and systems: Principles, characteristics, performance, and efficiency, IEEE Transactions on Applied Superconductivity, 26 (2016) 1-26.

[2] J.X. Jin, C. Grantham, Y.C. Guo, J.N. Li, S.X. Dou, Magnetic field properties of Bi2223/Ag HTS coil at $77 \mathrm{~K}$, Physica C Superconductivity, 278 (1997) 85-93.

[3] M.J. Wolf, W.H. Fietz, C.M. Bayer, S.I. Schlachter, R. Heller, K. Weiss, HTS CroCo: A stacked HTS conductor optimized for high currents and longlength production, IEEE Transactions on Applied Superconductivity, 26 (2016) 19-24.

[4] X. Liu, S. Wang, J. Qiu, J.G. Zhu, Y. Guo, Z.W. Lin, Robust optimization in HTS cable based on design for six sigma, IEEE Transactions on Magnetics, 44 (2008) 978-981.

[5] E.H. Brandt, M. Indenbom, Type-II-superconductor strip with current in a perpendicular magnetic field, Physical Review B, 48 (1993) 12893-12906.

[6] E.H. Brandt, M.V. Indenbom, A. Forkl, Type-II superconducting strip in perpendicular magnetic field, Europhysics Letters (EPL), 22 (1993) 735-740.

[7] Y. Mawatari, Critical state of periodically arranged superconducting-strip lines in perpendicular fields, Physical Review B, 54 (1996) 13215-13221.

[8] K.H. Müller, Self-field hysteresis loss in periodically arranged superconducting strips, Physica C: Superconductivity, 289 (1997) 123-130.

[9] A. Forkl, H. Kronmüller, A contribution to the analysis of the currentdensity distribution in elongated hard type-II superconductors with rectangular cross-section, Physica C: Superconductivity, 228 (1994) 1-14.

[10] T. Schuster, H. Kuhn, E.H. Brandt, M. Indenbom, M.R. Koblischka, M. Konczykowski, Flux motion in thin superconductors with inhomogeneous pinning, Physical Review B, 50 (1994) 16684-16707.

[11] Z.W. Lin, J.W. Cochrane, N.E. Lumpkin, G.J. Russell, Magneto-optical observations of magnetic flux distribution in a high-temperature superconductor x-array, Physica C: Superconductivity, 312 (1999) 247-254.

[12] R. Brambilla, F. Grilli, L. Martini, Development of an edge-element model for AC loss computation of high-temperature superconductors, Superconductor Science and Technology, 20 (2006) 16-24.

[13] B. Shen, F. Grilli, T. Coombs, Review of the AC loss computation for HTS using $\mathrm{H}$ formulation, Superconductor Science and Technology, 33 (2020) 033002.

[14] B. Shen, C. Li, J. Geng, Q. Dong, J. Ma, J. Gawith, K. Zhang, Z. Li, J. Chen, W. Zhou, X. Li, J. Sheng, Z. Li, Z. Huang, J. Yang, T.A. Coombs, Power dissipation in the HTS coated conductor tapes and coils under the action of different oscillating currents and fields, IEEE Transactions on Applied Superconductivity, 29 (2019) 1-5. 
[15] V.M.R. Zermeno, A.B. Abrahamsen, N. Mijatovic, B.B. Jensen, M.P. Sørensen, Calculation of alternating current losses in stacks and coils made of second generation high temperature superconducting tapes for large scale applications, Journal of Applied Physics, 114 (2013) 173901.

[16] L. Jiang, C. Xue, Y.-H. Zhou, Parallel magnetic field dependence of AC losses in periodically arranged superconductors with ferromagnetic substrates, Physica C: Superconductivity and its Applications, 566 (2019) 1353521.

[17] B. Shen, T. Coombs, F. Grilli, Investigation of AC loss in HTS crossconductor cables for electrical power transmission, IEEE Transactions on Applied Superconductivity, 29 (2019) 1-5.

[18] B. Shen, J. Li, J. Geng, L. Fu, X. Zhang, H. Zhang, C. Li, F. Grilli, T.A. Coombs, Investigation of AC losses in horizontally parallel HTS tapes, Superconductor Science and Technology, 30 (2017) 075006.

[19] G.G. Sotelo, M. Carrera, J. Lopez-Lopez, X. Granados, H-formulation FEM modeling of the current distribution in $2 \mathrm{G}$ HTS tapes and its experimental validation using Hall probe mapping, IEEE Transactions on Applied Superconductivity, 26 (2016) 1-10.

[20] Z. Wu, Y. Xue, J. Fang, L. Yin, D. Chen, The influence of the YBCO tape arrangement and gap between the two tapes on AC Loss, IEEE Transactions on Applied Superconductivity, 26 (2016) 1-5.

[21] A. Baskys, A. Patel, S.C. Hopkins, B.A. Glowacki, Modeling of trapped fields by stacked (RE)BCO tape using angular transversal field dependence, IEEE Transactions on Applied Superconductivity, 26 (2016) 1-4.

[22] A. Patel, S.C. Hopkins, B.A. Glowacki, Trapped fields up to $2 \mathrm{~T}$ in a 12 $\mathrm{mm}$ square stack of commercial superconducting tape using pulsed field magnetization, Superconductor Science and Technology, 26 (2013) 032001.

[23] K. Selva, G. Majkic, Trapped magnetic field profiles of arrays of (Gd,Y)Ba2Cu3Oxsuperconductor tape in different stacking configurations, Superconductor Science and Technology, 26 (2013) 115006.

[24] M. Zhang, W. Yuan, D.K. Hilton, M.D. Canassy, U.P. Trociewitz, Study of second-generation high-temperature superconducting magnets: the selffield screening effect, Superconductor Science and Technology, 27 (2014) 095010.

[25] L. Wang, J. Zheng, F. Jiang, R. Kang, Numerical simulation of AC loss in $2 \mathrm{G}$ high-temperature superconducting coils with $2 \mathrm{D}$-axisymmetric finite element model by magnetic field formulation module, Journal of Superconductivity and Novel Magnetism, 29 (2016) 2011-2018.

[26] B. Shen, F. Grilli, T. Coombs, Overview of H-Formulation: A versatile tool for modeling electromagnetics in high-temperature superconductor applications, IEEE Access, 8 (2020) 100403-100414.

[27] Z. Min, J.-H. Kim, S. Pamidi, M. Chudy, T.A. Coombs, Erratum: "Study of second generation, high temperature superconducting coils: Determination of critical current" [J. Appl. Phys. 111, 083902 (2012)], Journal of Applied Physics, 112 (2012) 083902.

[28] W.T. Norris, Calculation of hysteresis losses in hard superconductors carrying ac: Isolated conductors and edges of thin sheets, Journal of Physics D: Applied Physics, 3 (1970) 489-507. 